Research Article

\title{
The Effect of Solvent-Modification on the Physicochemical Properties of ZnO Nanoparticles Synthesized by Sol-Gel Method
}

\author{
Zainab Yousif Shnain*, Manal Afham Toma, Basheer A. Abdulhussein, \\ Najat J. Saleh, Mohammed Ibrahim, Nerran Manuel, Abbner Mahmood \\ Department of Chemical Engineering, University of Technology, Baghdad, Iraq
}

Received: $22^{\text {nd }}$ September 2021; Revised: $4^{\text {th }}$ November 2021; Accepted: $5^{\text {th }}$ November 2021 Available online: $9^{\text {th }}$ November 2021; Published regularly: March 2022

\section{Abstract}

This study investigated the solvent effect on the synthesis of Zinc Oxide ( $\mathrm{ZnO}$ ) nanoparticle using sol-gel method. Zinc acetate dihydrate and oxalic acid were used as a chemical precursor for the synthesis of the $\mathrm{ZnO}$ nanoparticle considering the effects of various solvents. The effect of using water, propanol, or ethanol as solvent during the synthesis were examined. The resultant gel in the aqueous and organic moderate solvent was thermally cracked into $\mathrm{ZnO}$ nanoparticles at $450{ }^{\circ} \mathrm{C}$ under atmospheric pressure. The results showed that the solvent type has a significant effect on the morphology and particles size of the $\mathrm{ZnO}$ nanoparticles synthesized. Atomic Force Microscopy (AFM) was used to investigate the microstructure of the nanoparticles. The crystalline and chemical structure of the prepared $\mathrm{ZnO}$ nanoparticle were studied by X-ray diffraction (XRD) and Fourier Transform Infrared spectroscopy (FTIR). The average diameter of nano-size particles obtained for ethanol, propanol and water are $79.55 \mathrm{~nm}$, $83.86 \mathrm{~nm}$ and $85.59 \mathrm{~nm}$, respectively. ZnO particles showed higher degree of crystalline in water compared to other solvents under current investigation.

Copyright (C) 2021 by Authors, Published by BCREC Group. This is an open access article under the CC BY-SA License (https://creativecommons.org/licenses/by-sa/4.0).

Keywords: ZnO Nanoparticle; Sol-gel; Organic Solvent; Semiconductors; Physicochemical properties

How to Cite: Z.Y. Shnain, M.A. Toma, B.A. Abdulhussein, N.J. Saleh, M. Ibrahim, N. Manuel, A. Mahmood (2022). The Effect of Solvent-Modification on the Physicochemical Properties of ZnO Nanoparticles Synthesized by Sol-Gel Method. Bulletin of Chemical Reaction Engineering \& Catalysis, 17(1), 46-52 (doi: 10.9767/bcrec.17.1.12345.46-52)

Permalink/DOI: https://doi.org/10.9767/bcrec.17.1.12345.46-52

\section{Introduction}

Recently, semiconductors materials are gaining relevance especially those prepared from metal oxides. The nanoscale level of semiconductors materials started to play important role for various industrial applications based on their unique properties, such as: suitable band gap, good thermal, and chemical stability [1]. The size of the materials is an important characteristic which is dependent on the optical and

* Corresponding Author.

Email: 80062@uotechnology.edu.iq;

zyousif.1973@gmail.com (Z.Y. Shnain) electrical properties otherwise called quantum confinement effects [2]. Zinc Oxide ( $\mathrm{ZnO})$ as a substantial semiconductor possess a band gap of $3.3 \mathrm{ev}$ at room temperature, large bond strength $(\mathrm{EX}=6 \mathrm{Mev})$ and high melting temperature (2248 K) [3].

Structural, optical and cathodoluminescence properties of undoped and Tin-doped $\mathrm{ZnO}$ thin films synthesized by spray pyrolysis has been reported by Karyaoui et al. [3]. Apart from this, $\mathrm{ZnO}$ has great photo stability and electrochemical coupling coefficient properties, Moreover, $\mathrm{ZnO}$ are highly stable and possesses various radiation absorptive ranges [4]. As a result of 
their non-toxicity, it serves as an environmentally friendly oxidizing agent with wide applications as ultraviolet (UV) absorptive in solar energy conversion and sun shelter [5]. Other areas of applications are in rubber additives, pigments gas sensors [6-9], photovoltaic devices, photo catalysis, clear controlling coating and electrostatic transverse [10-15]. In any given metal nanoparticles, their properties are a function of their method of synthesis. For the $\mathrm{ZnO}$, various approaches have been used to synthesize them, such as: arc discharge, chemical vapor condensation, hydrothermal, hydrogen plasma-metal reaction, micro emulsion, pyrolysis vapor phase, chemical reduction, sol gel and so on [16-18]. As a result of its ability to occur in various convoluted morphologies, it has various unique properties [9].

Hence, the various roles played by solvent in each reaction, the effects of solvent on the preparation of $\mathrm{ZnO}$ by sol-gel method has been studied. These solvent does not only influence the reaction, but also serve as a means to control the temperature in such a way to determine the peak temperature at which the reaction occur. This has necessitated the study of the influence of these solvent on the preparation of this zinc oxide. Different approaches have been used to prepare the $\mathrm{ZnO}$ through solgel approach $[19,20]$. This study therefore aimed to synthesize $\mathrm{ZnO}$ nanoparticles by Solgel method considering the effect of solventtype on its properties. The $\mathrm{ZnO}$ nanoparticle from the reaction between oxalic acid and zinc acetate using three different organic and aqueous solvents. Nano $\mathrm{ZnO}$ particles was obtained by thermal disintegration of organic and aqueous mediated $\mathrm{Zn}$ - oxalate at $450^{\circ} \mathrm{C}$. The Nanoparticles obtained were identified and characterized with Atomic Force Microscope (AFM), $\mathrm{X}$-ray Diffraction (XRD) and Fourier Infrared Spectrophotometer (FTIR).

\section{Materials and Methods}

\subsection{Materials and Characterization}

$$
\mathrm{Z} \text { in c a c e tat e dihydrate }
$$
$\left[\mathrm{Zn}\left(\mathrm{CH}_{3} \mathrm{COO}\right)_{2} .2 \mathrm{H}_{2} \mathrm{O}\right]$, Sodium Hydroxide $(\mathrm{NaOH})$, Ethanol $\left(\mathrm{C}_{2} \mathrm{H}_{6} \mathrm{O}\right)$, propanol $\left(\mathrm{C}_{3} \mathrm{H}_{8} \mathrm{O}\right)$ was purchased from Sigma-Aldrich and used without further purification. The structural, morphological and characteristics of $\mathrm{ZnO}$ nanoparticles were studied by means of $(\mathrm{Cu}-\mathrm{K} \alpha)$ XRD-D8 Advance Burker diffract meter, AFM (VEGA 3LM) and Fourier transformation infrared spectroscopy FTIR9 Perkin-Elmer Spectrum BX automatic FTIR spectrometer at 4 $\mathrm{cm}^{-1}$ resolutions.

\subsection{Synthesis of $\mathrm{ZnO}$ Nanoparticles}

The synthesis of the $\mathrm{ZnO}$ nanoparticles was carried out using sol-gel method. Three experiments were performed using three samples each with different solvent at the similar conditions. The first sample was prepared using water as a solvent and is referred to as $\mathrm{ZW}$, the second sample was prepared with propanol as a solvent and is referred to as $\mathrm{ZP}$ and the third sample was prepared with ethanol and is referred to as ZE. In each case, $2 \mathrm{~g}$ of zinc acetate dihydrate $\left[\mathrm{Zn}\left(\mathrm{CH}_{3} \mathrm{COO}\right)_{2} .2 \mathrm{H}_{2} \mathrm{O}\right]$ was dissolved in $200 \mathrm{~mL}$ of solvent by continuous stirring for $2 \mathrm{~h}$ until full dissolution and clear solutions were obtained. $1.0 \mathrm{M}$ of oxalic acid was later titrated into the prepared solution until it reaches the $\mathrm{pH}$ of 9 and a homogeneous milky slurry solution has been formed after $1.5 \mathrm{~h}$. Then the sample was left for a period of $24 \mathrm{~h}$ hat room temperature in order to obtain a gelatin form and complete hydrolysis. Then the samples were filtrated, dried in oven, and grounded by ball mill. The samples prepared were heat treated at $450{ }^{\circ} \mathrm{C}$ for $2 \mathrm{~h}$, cooled and labeled as $\mathrm{ZP}, \mathrm{ZE}$, and $\mathrm{ZW}$.

\subsection{Characterization of the $\mathrm{ZnO}$ Nanoparticles}

The crystallite size of the $\mathrm{ZnO}$ nanoparticle as well as its crystal-phase structure were studied using X-ray diffractometer. Scherrer Equation (1) was used to calculate the $\mathrm{ZnO} \mathrm{NP}$ crystallite size as summarized in Table 1. AFM was used to further obtain information on the size distribution and average particle size of the prepared $\mathrm{ZnO}$ nanoparticles.

$$
D=\frac{k \lambda}{\beta \cos \theta}
$$

From the equation (1), $k$ denotes the Scherer constant of a value of $0.89, \lambda, \theta$ and $\beta$ are the $\mathrm{X}$ ray wavelength, Bragg diffraction angle and the peak width of the half maximum, respectively.

Table 1 . The crystallite size of $\mathrm{ZnO}$ nanoparticles prepared with different solvents.

\begin{tabular}{cc}
\hline Samples & Average crystallite size $(\mathrm{nm})$ \\
\hline ZW & 34.16 \\
ZP & 30.65 \\
ZE & 22.65 \\
\hline
\end{tabular}




\section{Results and Discussion}

The yield percent of the final $\mathrm{ZnO}$ nanoparticles obtained from the different solvents was determined gravimetrically and it was estimated as $90 \%$ for the three solvents which is consistent with the that obtained by Kanade et al. [19]. The reaction leading to the formation of the $\mathrm{ZnO}$ occurs in alkyl and aqueous media and is depicted in Equation (2).

$$
\begin{aligned}
& \mathrm{Zn}\left(\mathrm{CH}_{3} \mathrm{COO}\right)_{2} \cdot 2 \mathrm{H}_{2} \mathrm{O}+\mathrm{H}_{2} \mathrm{C}_{2} \mathrm{O}_{4} \cdot 2 \mathrm{H}_{2} \mathrm{O} \\
& \quad \rightarrow \mathrm{ZnC} \mathrm{C}_{2} \mathrm{O}_{4} \cdot 2 \mathrm{H}_{2} \mathrm{O}+2 \mathrm{CH}_{3} \mathrm{COOH}+2 \mathrm{H}_{2} \mathrm{O}
\end{aligned}
$$

It was observed that the reaction was quicker in aqueous medium than in alkyl solvent, which could be ascribed to the great polarity and dielectric constant of water as contrast to the alkyl correspondent parts. This reaction is slow and displayed a homogenous nucleation in alkyl solvent. However, the ethanol and propanol can be performed as a great dissipate solvent through the reaction. The alkyl solvents played significant role in controlling the crystal growth [20].

The characterizations of the $\mathrm{ZnO}$ showing the XRD patterns, designated as ZW, ZE, and $\mathrm{ZP}$, are depicted in Figures 1(a), (b) and (c). Figure 1(a) shows the XRD pattern of the ZW prepared using water as the solvent. The peaks
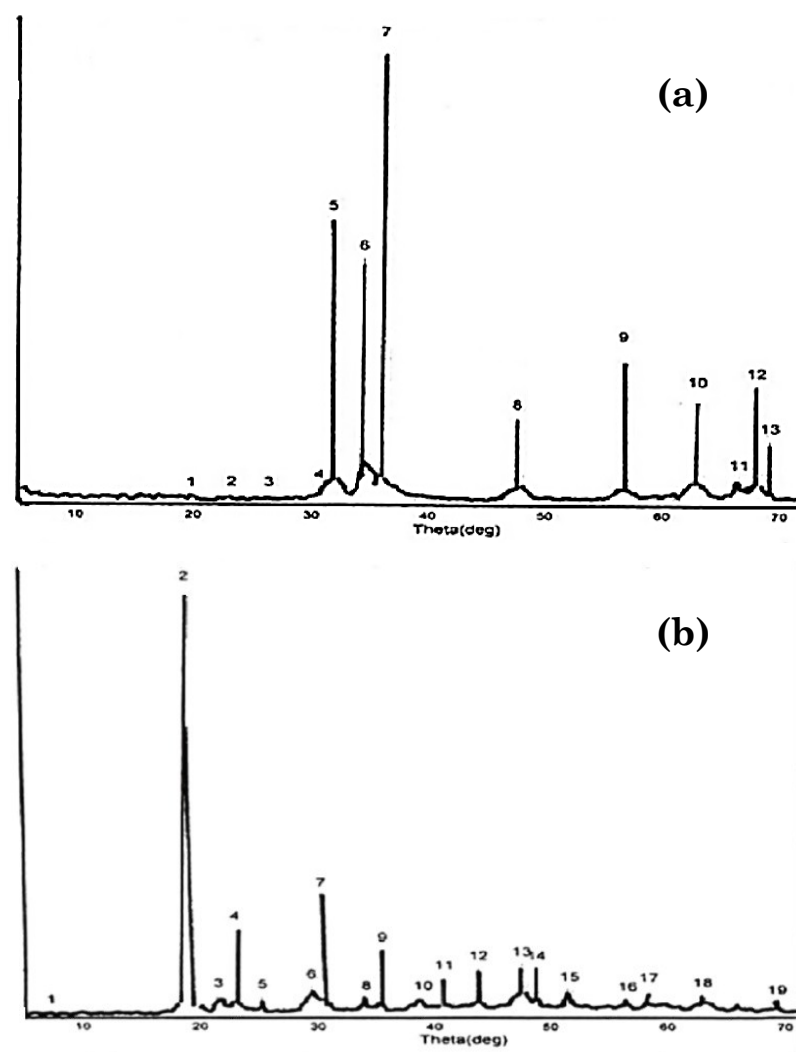

represented by $5,6,7,8,9,10,11,12$, and 13 corresponds to the diffraction lines (100), (002), (101), (102), (110), (103), (200), (112), and (201), respectively. No peak of impurities was observed for water samples synthesized in an aqueous medium. Figure 1(b) display the XRD pattern of ZE sample prepared using ethanol as solvent. The peaks of the $\mathrm{ZnO}$ at $7,8,9,14$, 16 and 19 corresponds to the diffraction lines (100), (002), (101), (102), (110) and (103), respectively. However, other observed peaks are due to the presence of impurities. Figure $1(\mathrm{c})$, represents the XRD pattern of the ZP sample prepared using propanol as solvent. The peaks of the $\mathrm{ZnO}$ are $9,10,11,12,16$, and 20 which denotes the diffraction lines at (100), (002), (101), (102), (110), and (103), respectively. The unidentified peaks can be attributed to the presence of impurities. The impurities observed in ZP and ZE can be assigned to the unreacted zinc acetate. From the morphologies shown in Figures (1b) and (c) using ethanol and propanol as solvent, it can be observed that the diffraction patterns are very similar to each one another. Whereas the XRD pattern of the ZW sample prepared using water shows a structure of $\alpha-\mathrm{ZnC}_{2} \mathrm{O}_{4} \cdot 2 \mathrm{H}_{2} \mathrm{O}$ corresponding to the peak at $2 \theta=19.5^{\circ}$ and the formation of the structure $\beta-\mathrm{ZnC}_{2} \mathrm{O}_{4} \cdot 2 \mathrm{H}_{2} \mathrm{O}$ at $2 \theta=24^{\circ}$. Both structures are unobserved with ethanol and propanol. The XRD pattern of the ZW prepared using water shows a hexagonal phase of $\mathrm{ZnO}$ with a clear intense and sharp peak compared with other solvents which indicate the formation of high crystallinity of $\mathrm{ZnO}$ nanostructure.

Table 1 shows the values of the calculated average crystallite sizes of all the $\mathrm{ZnO}$ samples using Scherrers equation. The results were obtained when half maximum of the x-ray diffraction peaks, and their full width were used. From the result, the crystallite size of the sam-

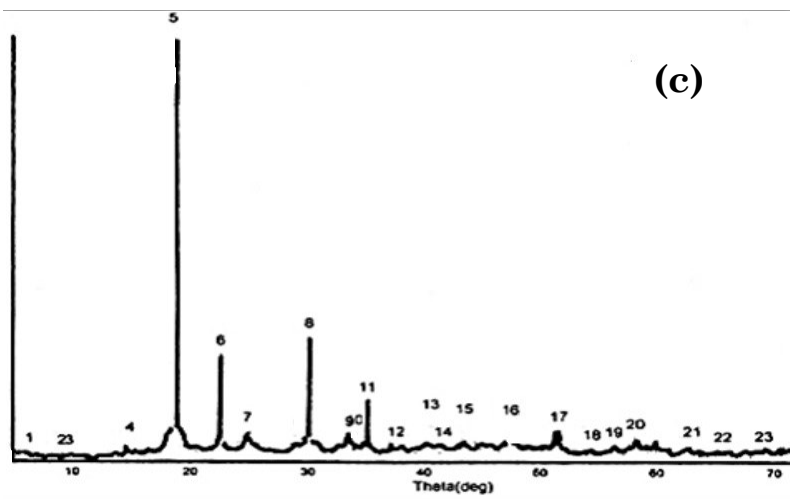

Figure 1. XRD patterns of (a) ZW sample prepared using water (b) ZE sample prepared using ethanol (c) ZP sample prepared using propanol. 


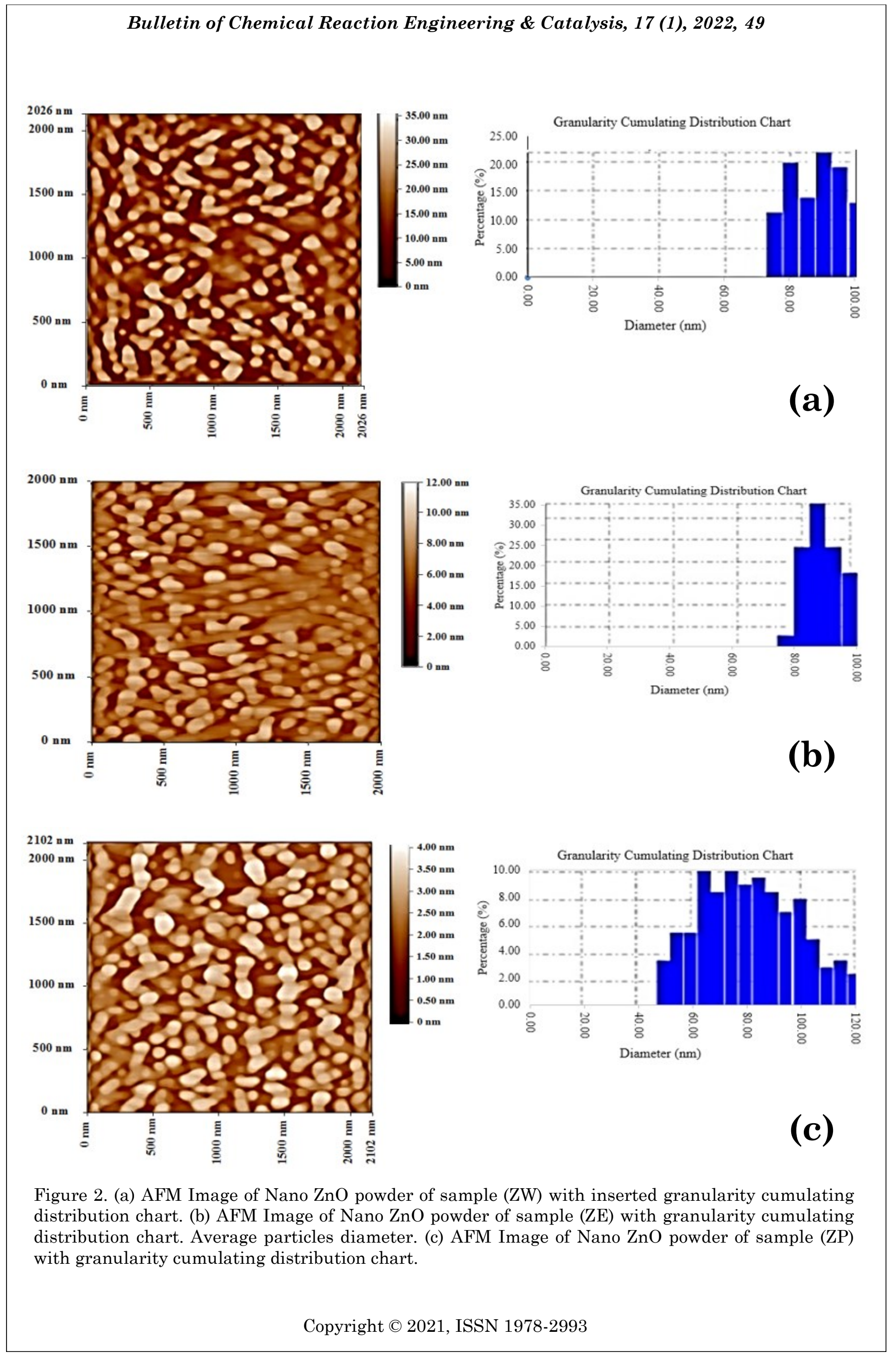


ples synthesized using water as solvent displayed the high purity of the prepared $\mathrm{ZnO}$. The estimated crystallite size of zinc oxide powders prepared with various solvents are consistent with that reported by previous researchers [21].

Figures 2 (a), (b), and (c) show the AFM results of the ZW, ZP and ZE, respectively. The AFM were conducted to study the microstructure homogeneities and morphologies for $\mathrm{ZnO}$ nanoparticles. Figure 2(a) represents AFM results for aqueous mediated $\mathrm{ZnO}$ nanoparticles. Meanwhile, Figures 2(b) and (c) display organic mediated $\mathrm{ZnO}$ nanoparticles of: ethanol and the propanol respectively, mixtures of bar and spheres particle shape were obtained for all sample. However, it is interesting to note that the nanoparticles in these samples tend to agglomerate due to their tiny structures and sizes. Such agglomeration could be attributed to the extended reaction time. However, the agglomeration in the aqueous medium tends to be more obvious compared to their organic solvent counterpart [16]. This could be due to the fact that organic solvents possessing higher dispersing capability [22]. To prevent agglomeration, the presence of alkyl molecule introduced steric hindrance between the particles of the $\mathrm{ZnO}$ [23], consequently, the particles depict varying particles sizes and morphology with respect to the solvent use. The particle size of the $\mathrm{ZnO}$ prepared in alkyl mediate solvent are smaller
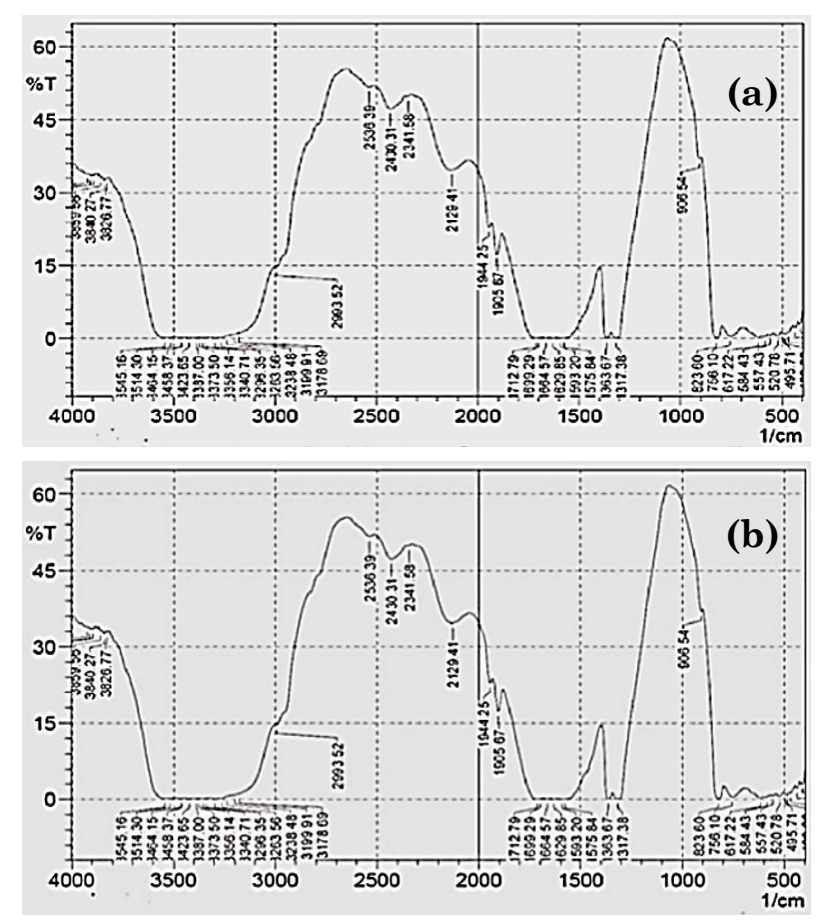

compared to that of the water. In addition to this, they exhibit different morphology. Their values are 79.55 and $83.86 \mathrm{~nm}$ for ethanol and propanol while that of the water is $85.59 \mathrm{~nm}$. The observed smaller particles size obtained for these organic solvents could be attributed to the oxalates used in the synthesis of the oxide in the various media. The lower particle size and different morphology shows that alkyl containing solvents are acting as a master role in controlling the seeding and crystal adjustment [24]. The nano-size particles attained with propanol mediated samples are greatest excessivefine attributable to its superior scattering performance in comprise to corresponding parts [25].

The FTIR analysis of the three $\mathrm{ZnO}$ samples prepared using water, ethanol and propanol are depicted in Figures 3 (a), (b) and (c). The infrared band of Zn-O bond corresponding to bulk properties can be seen at wavelength of $580 \mathrm{~cm}^{-1}$. Moreover, a shift in peak can be observed at wavelength of 375 and $419 \mathrm{~cm}^{-1}$ with respect to the water and alkyl solvents respectively. This signify that the role of solvent on the preparation and formation of nanosized $\mathrm{ZnO}$ particles. Furthermore, the tiny particles lost the elongated range order, thus adopts the localize pattern of octahedral symmetry. The vacancy of stretching band owing to oxalate and acetate confirm complete creation of $\mathrm{ZnO}$ [26,27].

\section{Conclusion}

In this paper $\mathrm{ZnO}$ nano-sized powder was synthesis using oxalic acid, zinc acetate, and zinc oxalate as precursor. The effects of utilizing three different solvents namely water, ethanol and propanol as solvents on the properties of $\mathrm{ZnO}$ was also investigated. The hexagonal crystal structure of the $\mathrm{ZnO}$ was confirmed by XRD analysis. The average diameter of the

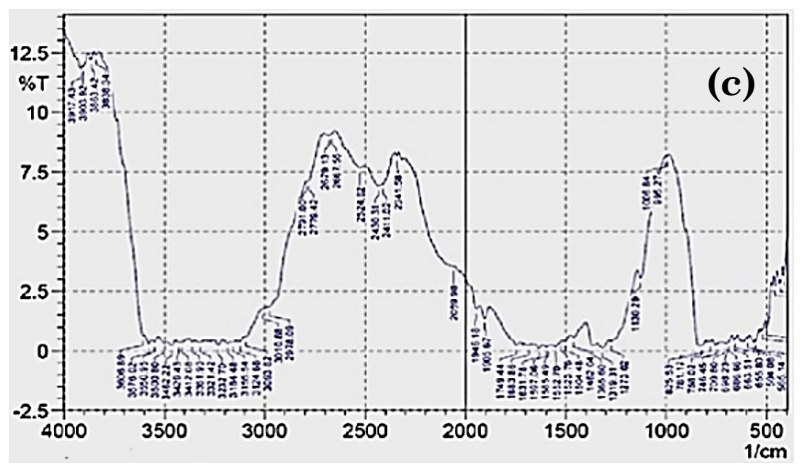

Figure 3. (a) FTIR OF ZW sample prepared used water solvent. (b) FTIR OF ZW sample prepared used ethanol solvent. (c) FTIR of ZP sample prepared used zinc acetate propanol solvent. 
$\mathrm{ZnO}$ particles were obtained as 79.55 and 83.86 $\mathrm{nm}$ for $\mathrm{ZnO}$ prepared using ethanol and propanol, respectively, while $85.59 \mathrm{~nm}$ was obtained for $\mathrm{ZnO}$ prepared using water. The water mediated non-reaction in the ethanol and propanol leads to the formation of a bars and spheres mixture. When water was used as a solvent, highly crystalline nanoparticles $\mathrm{ZnO}$ was obtained due to the higher crystallite size as calculated by Scherrer's equation and observed by XRD pattern. Both nucleation and growth of $\mathrm{ZnO}$ nano-sized particles are affected by the polarity of the solvent thus affecting the shape and the size of $\mathrm{ZnO}$ obtained.

\section{Acknowledgement}

Authors acknowledge the support of Department of Chemical Engineering, University of Technology, Iraq.

\section{References}

[1] Fan, D., Afzaal, M., Mallik, M.A., Nguyen, C.Q., O’Brien, P., Thomas, P.J. (2007). Using coordination chemistry to develop new routes to semiconductor and other materials. Coordination Chemistry Reviews, 251(13-14), 18781888. DOI: 10.1016/j.ccr.2007.03.021

[2] Malik, M.A., Afzaal, M., O’Brien, P. (2010). Precursor chemistry for main group elements in semiconducting materials. Chemical Reviews, 110(7), 4417-4446. DOI: $10.1021 /$ cr900406f

[3] Gonzalez-Hernandez, R., Martinez, A.I., Falcony, C., Lopez, A.A., Pech-Canul, M.I., HdzGarcia, H.M. (2010). Study of the properties of undoped and fluorine doped zinc oxide nanoparticles. Materials Letters, 64(13), 14931495. DOI: 10.1016/j.matlet.2010.04.001

[4] Kołodziejczak-Radzimska, A., Jesionowski, T. (2014). Zinc oxide-from synthesis to application: a review. Materials, 7(4), 2833-288. DOI: 10.3390/ma7042833

[5] Becheri, A. (2008). Synthesis and characterization of zinc oxide nanoparticles: application to textiles as UV-absorbers, Journal of Nanoparticle Research, 10(4) 679-689. DOI: 10.1007/s11051-007-9318-3

[6] Giovannelli, F., Bah, M., Delorme, F., MonotLaffez, I. (2021). Influence of composition on morphology of semiconducting oxides microwires. Journal of Physics and Chemistry of Solids, $158, \quad 110248$. D O I : 10.1016/j.jpcs.2021.110248
[7] Fu, Y.S., Du, X.W., Kulinich, S.A. (2007). Stable aqueous dispersion of $\mathrm{ZnO}$ quantum dots with strong blue emission via simple solution route, Journal of the American Chemical Society, 129(51), 16029-16033. DOI: 10.1021/ja075604i

[8] Karyaoui, M., Jemia, D.B., Gannouni, M., Assaker, I.B., Bardaoui, A., Amlouk, M., Chtourou, R. (2020). Characterization of Agdoped $\mathrm{ZnO}$ thin films by spray pyrolysis and its using in enhanced photoelectrochemical performances. Inorganic Chemistry Communications, 119, 108114 . DOI: 10.1016/j.inoche.2020.108114

[9] Marzec, A., Szadkowski, B., Rogowski, J., Rybiński, P., Maniukiewicz, W. (2021). Novel eco-friendly hybrid pigment with improved stability as a multifunctional additive for elastomer composites with reduced flammability and $\mathrm{pH}$ sensing properties. Dyes and Pigments, 186, 108965 . DOI: 10.1016/j.dyepig.2020.108965

[10] Zhang, Q., Xu, M., You, B., Zhang, Q., Yuan, H., Ostrikov, K.K. (2018). Oxygen vacancy mediated $\mathrm{ZnO}$ nanoparticle photocatalyst for degradation of methylene blue. Applied Sciences, 8(3), 353. DOI: 10.3390/app8030353

[11] Roy, N., Chakraborty, S. (2021). ZnO as photocatalyst: An approach to waste water treatment. Materials Today: Proceedings, 46, 6399-6403. DOI: 10.1016/j.matpr.2020.06.264

[12] Byzynski, G., Pereira, A.P., Volanti, D.P., Ribeiro, C., Longo, E. (2018). High-performance ultraviolet-visible driven $\mathrm{ZnO}$ morphologies photocatalyst obtained by microwave-assisted hydrothermal method. Journal of Photochemistry and Photobiology A: Chemistry, 353,

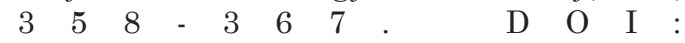
10.1016/j.jphotochem.2017.11.032

[13] Quiñones, R., Shoup, D., Behnke, G., Peck, C., Agarwal, S., Gupta, R.K., Wang, Q. (2017). Study of perfluorophosphonic acid surface modifications on zinc oxide nanoparticles. Materials, 10(12), 1363 . DOI: 10.3390/ma10121363

[14] Kulinich, Y.S. (2007). Stable aqueous dispersion of $\mathrm{ZnO}$ quantum dots with strong blue emission via simple solution route. Journal of the American Chemical Society, 129(51), 16029-16033. DOI: 10.1021/ja075604i

[15] Tonto, P., Mekasuwandumrong, O., Phatanasri, S., Pavarajarn, V., Praserthdam, P. (2008) Preparation of $\mathrm{ZnO}$ nanorod by solvothermal reaction of zinc acetate in various alcohols. Ceramics International, 34(1), 5762. DOI: 10.1016/j.ceramint.2006.08.003 
[16] Harun, K., Mansor, N., Ahmad, Z.A., Mohamad, A.A. (2016). Electronic properties of $\mathrm{ZnO}$ nanoparticles synthesized by Sol-gel method: a LDA+ U calculation and experimental study. Procedia Chemistry, 19, 125-132. DOI: 10.1016/j.proche.2016.03.125

[17] Mohan, A.C., Renjanadevi, B. (2016). Preparation of zinc oxide nanoparticles and its characterization using scanning electron microscopy (SEM) and X-ray diffraction (XRD). Procedia Technology, 24, 761-766. DOI: 10.1016/j.protcy.2016.05.078

[18] Hasnidawani, J.N., Azlina, H.N., Norita, H., Bonnia, N.N., Ratim, S., Ali, E.S. (2016). Synthesis of $\mathrm{ZnO}$ nanostructures using sol-gel method. Procedia Chemistry, 19, 211-216. DOI: 10.1016/j.proche.2016.03.095

[19] Hasanpoor, M., Aliofkhazraei, M., Delavari, H. (2015). Microwave-assisted synthesis of zinc oxide nanoparticles. Procedia Materials Science, $\quad 11, \quad 320-325$. DO I: 10.1016/j.mspro.2015.11.101

[20] Kanade, K.G., Kale, B.B., Aiyer, R.C., Das, B.K. (2006). Effect of solvents on the synthesis of nano-size zinc oxide and its properties. Materials Research Bulletin, 41(3), 590-600. DOI: 10.1016/j.materresbull.2005.09.002

[21] Xu, H., Wang, H., Zhang, Y., He, W., Zhu, M., Wang, B., Yan, H. (2004). Hydrothermal synthesis of zinc oxide powders with controllable morphology. Ceramics International, 30(1), 93-97. DOI: 10.1016/S0272-8842(03)00069-5

[22] Chandra, S., Karak, N. (2018). Environmentally friendly polyurethane dispersion derived from dimer acid and citric acid. ACS Sustainable Chemistry \& Engineering, 6(12), 16412 16423. DOI: 10.1021/acssuschemeng.8b03474
[23] Quiñones, R., Shoup, D., Behnke, G., Peck, C., Agarwal, S., Gupta, R.K., Wang, Q. (2017). Study of perfluorophosphonic acid surface modifications on zinc oxide nanoparticles. Materials, 10(12), 1363. DOI: 10.3390/ma10121363

[24] Kannan, S., Sekar, A., Sivaperuman, K. (2020). Effects of the molecular structure on the second-order nonlinear optical properties of stilbazolium derivative single crystals: a review. Journal of Materials Chemistry $C$, $8(47), \quad 16668-16690$. D O I : 10.1039/D0TC04260A

[25] Qin, H., Guo, W., Liu, J., Xiao, H. (2019). Size-controlled synthesis of spherical $\mathrm{ZrO} 2$ nanoparticles by reverse micelles-mediated sol-gel process. Journal of the European Ceramic Society, 39(13), 3821-3829. DOI: 10.1016/j.jeurceramsoc.2019.04.035

[26] Jaafar, N.F., Najman, A.M.M., Marfur, A., Jusoh, N.W.C. (2020). Strategies for the formation of oxygen vacancies in zinc oxide nanoparticles used for photocatalytic degradation of phenol under visible light irradiation. Journal of Photochemistry and Photobiology A: Chemistry, 388, 112202. DOI: 10.1016/j.jphotochem.2019.112202

[27] Shnain, Z.Y., Ali, J.M., Sukkar, K.A., Alsaffar, M.A., Abid, M.F. (2021). A computational fluid dynamics study of liquid-solid nanofluid flow in horizontal pipe. Arabian Journal for Science and Engineering, 1-10. DOI: 10.1007/s13369-021-05512-y 